\title{
ON THE IMPEDANCE OF THE SLICON DIOXIDE/ELECTROLYTE INTERFACE
}

\author{
LUC BOUSSE and PIEI BERGVELD. \\ Department of Electrical Engineenng. Twente Unroersity of Technology, $P O$ Box 217 , \\ 7500 AE Enschede (The Netherlands)
}

(Recelved 15th November 1982, in revised form 20th January 1983)

\begin{abstract}
The small-signal impedance of electrolyte/insulator/silicon structures is partly determined by the properties of the insulator/electrolyte interface A theoretical model for this interfacial impedance is derived. Two parallel contrnbutions are involved: the double-layer capacitance for which a Gouy-Chapman-Stern model is adopted, and a branch contauning the capactance related to the surface reactions with $\mathrm{H}^{+}$and $\mathrm{OH}^{-}$lons from the electrolyte These surface reactions cause the total interfacial impedance to be very low for insulators with a high surface reactinty such as, for instance, $\mathrm{Al}_{2} \mathrm{O}_{3}$ or $\mathrm{Ta}_{2} \mathrm{O}_{5}$ - For $\mathrm{SiO}_{2}$ surfaces, the reactuvity is much lower, implying a larger interfacial impedance. Measurements of the unterfacial impedance were carned out at low frequencies on $12 \mathrm{~nm} \mathrm{SiO}$ layers in $\mathrm{NaCl}_{2}$ electrolytes at Ionic strengths of $10^{-4}, 10^{-3}$ and $10^{-2} \mathrm{M}$. The results agreed with the theoretucal predictions which were based on parameter values obtamed from independent measurements of $\psi_{0} / \mathrm{pH}$ characteristics. The agreement confirms the model for the formation of the surface charge through reactions of fixed silanol groups in the $\mathrm{SiO}_{2}$ surface
\end{abstract}

\section{INTRODUCTION}

Direct experumental determination of the impedance of interfaces between an electrolyte solution and another phase are well known for the metal/electrolyte (for a recent review, see ref. 1) and the silver-iodide/electrolyte interface [2]. In these cases, the measurements can be performed because both sides of the interface are conducting for the ac signals used. Most of the present knowledge of the electrical double layer stems from the study of these two systems. An important aspect of the silver iodide system is that its double-layer properties have been studied in two different ways: the measurement of charge on colloid suspensions [3], from which the double layer capacitance can be derived, and durect measurement of this capacitance with Ag/AgI solid electrodes, which confirms the value derived by the indirect method [2].

Such a confirmation does not yet exist for the $\mathrm{SiO}_{2} /$ electrolyte interface where $\mathrm{H}^{+}$and $\mathrm{OH}^{-}$ions are potential determining ions (pdi). The only values of the double-layer capacitance on $\mathrm{SiO}_{2}$ mentioned in the literature have been calculated 
assuming that the Nernstian relation between $\mathrm{pH}$ variations and variations in surface potential is valid [4]. Ths assumption leads to low values of the capacitance around $\mathrm{pH}_{\mathrm{pzc}}$, whle at the same time doubt began to emerge concerning the validity of the Nernst equation [5-7]. To overcome this problem, Yates et al. proposed the site-bindıng theory [8], which has been successful in explaining the charge $/ \mathrm{pH}$ and the zeta potential/pH characteristics of oxide surfaces. This theory has also provided an explanation for the operation of electrolyte/insulator/sulicon (EIS) structurcs, and in particular ion-sensitive field effect transistors (ISFETs) [9-11]. Results obtained on structures of this type have shown directly that Nernst's equation cannot be applied to insulator/electrolyte interfaces [12].

Apart from the desire to obtain direct information about the impedance of the $\mathrm{S}_{1} \mathrm{O}_{2}$ / electrolyte interface, this impedance is important in the charactenzation of EIS structures. A number of studies of the influence of the electrolyte on surface states at the $\mathrm{S}_{1} \mathrm{O}_{2} / \mathrm{Si}$ interface have appeared [13.14], but the influence of the $\mathrm{S}_{1} \mathrm{O}_{2} /$ electrolyte impedance on the measurement method was not precisely known

We will therefore present impedance measurements on EIS structures where the insulator is a very thin $\mathrm{SiO}_{2}$ layer. Since this $\mathrm{SiO}_{2}$ layer forms a capacitance which is in senes with the double layer, a reference measurement in an electrolyte where the double layer has a negligible influence is required to obtain double-layer capacitances. To explain the results of these measurements, a theory of the insulator/ electrolyte impedance will be presented which is based on the general outline given by Sparnaay [15], who suggested that surface acid-base reactions such as described by Payens [16] could be applied to this problem.

\section{THE IMPEDANCE OF EIS STRUCTURES}

Although experımental data will only be presented for $\mathrm{S}_{1} \mathrm{O}_{2}$ surfaces, this section will discuss the impedance of EIS structures in general. This makes it possible to discuss the influence of the properties of any insulator on this impedance.

Conceptually speaking, EIS structures can be derived from the metal/insulator/ silicon (MIS) system by inserting an electrolyte solution between the metal (which then becomes a reference electrode) and the insulator. The simularity between these structures is important. because MIS properties have been intensely studied in silicon device physics. For both types of structure, the capacitance is the most important measurable parameter. In the case of an MIS structure, it is sufficient to consider an infinitesimal variation of the charge on the metal gate and the substrate in relation to the resulting change in potential. An EIS structure, as opposed to MIS structures, contains three vanable charges: the sulicon surface charge $Q_{5}$, the insulator surface charge $\sigma_{0}$, and the electrolyte diffuse layer charge $\sigma_{\mathrm{d}}$. (All charges and capacitances are implicitly considered to be expressed per unit area.) On addition to these vanable charges, the insulator itself contains a fixed charge. The total charge inside the insulator can be separated into the following components [17]-

$Q_{\mathrm{rot}}=Q_{\mathrm{ss}}+Q_{\mathrm{m}}$ 


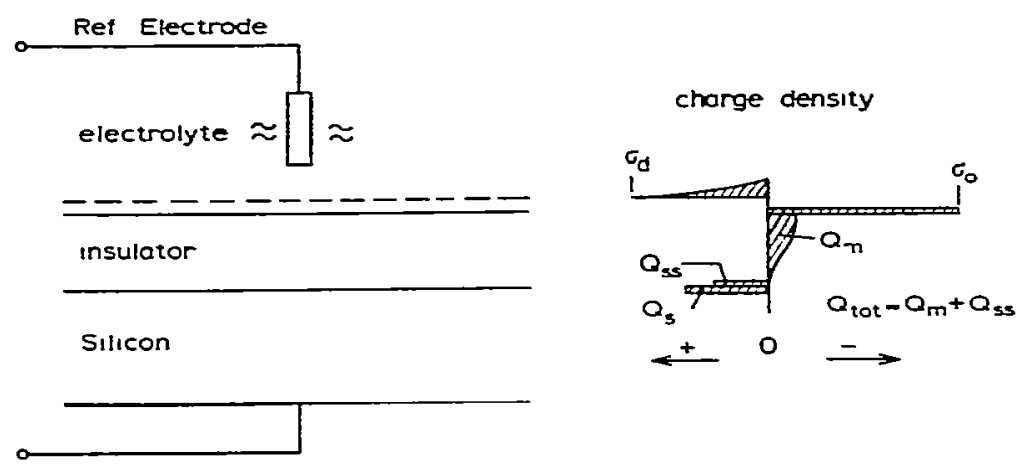

Fig. 1. Schematic representauon of a EIS structure together with possible charge distribution Dimensions and charges are not to scale The charges are represented for a case where $\mathrm{pH}>\mathrm{pH}_{\mathrm{pxc}}$ and the p-type substrate is biased in accumulation

where $Q_{s s}$ is the charge at the slicon/insulator interface (in practice almost always an $\mathrm{Si} / \mathrm{SiO}_{2}$ interface) including the charge localized in surface states at this interface, and $Q_{\mathrm{m}}$ represents the total charge distributed inside the insulator. Apart from the contribution of mobile alkali ions, this includes any charge introduced inside the insulator near the interface with the electrolyte. A representation of the structure and the charges in it is given in Fig. 1. The charge balance equation for the whole structure is

$Q_{\mathrm{s}}+Q_{\mathrm{tot}}+\sigma_{0}+\sigma_{\mathrm{d}}=0$

In the definition of capacitance, the incremental charge $d Q$ supplied by the external circuit 1s, for a non-conducting insulator,

$\mathrm{d} Q=\mathrm{d} Q_{\mathrm{s}}=-\left(\mathrm{d} \sigma_{0}+\mathrm{d} \sigma_{d}\right)$

The distribution of $\mathrm{d} Q$ between $\mathrm{d} \sigma_{0}$ and $\mathrm{d} \sigma_{\mathrm{d}}$ depends on the surface reactions taking place at the insulator/electrolyte interface, and this will be considered later.

Equation (3) contains the assumption that the insulator charge $Q_{\text {tot }}$ is not influenced by the incremental variations of charge and potential.

The necessary starting-point for the calculation of the total impedance is the expression of the voltage distributions across the EIS structure. This can be conveniently characterized by the flat-band voltage, which has been derived elsewhere [18]

$V_{\mathrm{FB}}=E_{\mathrm{ref}}-\frac{1}{q} \Phi^{\mathrm{st}}-\psi_{0}-Q_{\mathrm{t}} / C_{1}+\chi^{\mathrm{sel}}+\delta \chi$

where $E_{\text {ref }}$ is the reference electrode potential, $\Phi^{S_{1}}$ the work function of silicon, $\psi_{0}$ the potential drop in the electrolyte at the insulator/electrolyte interface, $\chi^{\text {Sol }}$ the surface dipole potential of the electrolyte, $\delta \chi$ collects a number of variations of $\chi$ potentials [18] and $C_{1}$ is the insulator capacitance. The $Q$, present in this equation is 
not the same as the total insulator charge $Q_{\text {tor }}$. To obtain the influence on the flat-band voltage, the charge at a location inside the insulator must be weighted by the distance to the insulator/electrolyte interface. Here, $Q_{1}$ can be written

$Q_{1}=Q_{s s}+\left(d_{c} / d_{1}\right) Q_{m}$

where $d_{c}$ is the distance of the centroid of the charge distribution $Q_{m}$ to the insulator/electrolyte interface. The charge located near this interface will have a much smaller effect on $V_{\mathrm{FB}}$ than on the global charge balance, according to eqns. (1) and (5). The existence of such a charge in $\mathrm{SiO}_{2}$ is probable, since it is known that $V_{\mathrm{FB}}$ drifts slowly with time for $\mathrm{S}_{1} \mathrm{O}_{2}$ insulators [19], a fact which cannot be explained by the fast reactions at the surface with $\mathrm{H}^{+} / \mathrm{OH}^{-}$ions, nor by variations of the surface-state density [14].

The general expression of the voltage applied to an EIS structure is [18]

$V_{\mathrm{a}}=V_{\mathrm{FB}}+\psi_{\mathrm{s}}-Q_{\mathrm{s}} / C_{\mathrm{I}}$

with $\psi_{\mathrm{s}}$ and $Q_{\mathrm{s}}$ the silicon surface potential and charge respectively. The impedance of the structure then follows from eqns. (3), (4) and (6):

$Z=-(J \omega)^{-1} \frac{\Delta V_{\mathrm{a}}}{\Delta Q_{s}}=(J \omega)^{-1}\left\{C_{s}^{-1}+C_{1}^{-1}+\frac{\Delta \psi_{0}}{\Delta Q_{s}}\right\}$

where $\omega$ is the angular frequency, and $J^{2}=-1$. The symbol $\Delta$ designates a small-signal sinusordal variation including phase and is, in general, complex. Equation (7) is represented in Fig. 2a. The term $C_{s}^{-1}=-\Delta \psi_{s} / \Delta Q_{s}$ is due to the silicon surface capacitance, which has been extensively studied [20] In particular, it is known that
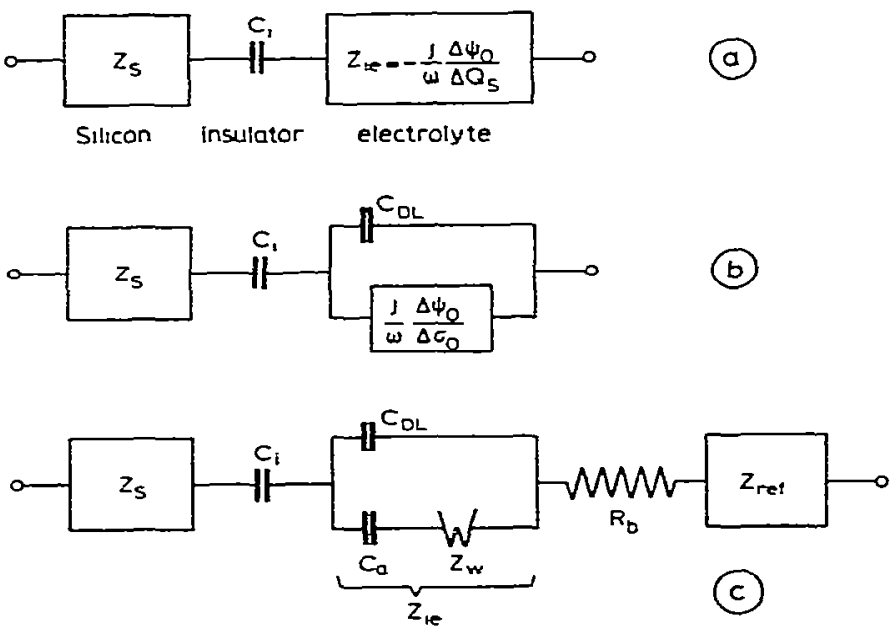

Fig 2 Successive stages in the development of the complete equivalent arcuit of an electrolyte/ insulator/silicon structure, $R_{\mathrm{b}}$ is the electrolyte bulk resistance and $Z_{\text {ret }}$ the reference electrode impedance 
the inversion-layer response and surface-state time constants introduce resistive components. Therefore, $C_{s}$ is not a pure capacitance but rather an impedance $Z_{s}$. The third term in eqn. (7) is caused by the insulator/electrolyte interface, whose impedance will be termed $Z_{1 e}$. Using eqn. (3), we may write

$Z_{1 \mathrm{e}}^{-1}=J \omega \Delta Q_{\mathrm{s}} / \Delta \psi_{0}=-j \omega\left(\frac{\Delta \sigma_{\mathrm{d}}}{\Delta \psi_{\mathrm{o}}}+\frac{\Delta \sigma_{0}}{\Delta \psi_{\mathrm{o}}}\right)$

Thus relation shows that the impedance introduced by this interface is composed of two parallel components. The furst of these originates from the capacitance of the electrical double layer, and is purely electrostatic in origin. For this capacitance we will adopt the Gouy-Chapman-Stern theory of the double layer, neglecting the possibility of adsorption of counterions in the compact part of the double layer. The charge in the diffuse part of the double layer in the case of a $1: 1$ symmetrical electrolyte is

$\sigma_{\mathrm{d}}=-\left(8 \epsilon_{\mathrm{w}} k T c\right)^{1 / 2} \sinh \left(q \psi_{\mathrm{d}} / 2 k T\right)$

and the total interface potential is

$\psi_{0}=\psi_{\mathrm{d}}-\sigma_{\mathrm{d}} / C_{\text {stern }}$

In these equations, $\psi_{\mathrm{d}}$ is the potential over the diffuse charge layer, $\epsilon_{\mathrm{w}}$ the dielectric constant of water, $c$ the electrolyte concentration in ion pairs per unit volume, $C_{\text {stern }}$ the compact layer capacitance and the other symbols have their usual meaning. The total double-layer capacitance $C_{\mathrm{DL}}=-\mathrm{d} \sigma_{\mathrm{d}} / \mathrm{d} \psi_{0}$ is the series combination of the Stern layer capacitance $C_{\text {stern }}$, and the capacitance of the Gouy-Chapman diffuse charge layer which is obtained by taking the derivative of eqn. (9). This last capacitance is potential dependent, and can be expressed either in terms of $\psi_{d}$ or of $\sigma_{\mathrm{a}}$ In general, the former is preferred, giving

$C_{\mathrm{DL}}^{-1}=C_{\text {stern }}^{-1}+\left\{q\left(2 \epsilon_{w} k T c\right)^{1 / 2} / k T \cosh \left(q \psi_{\mathrm{d}} / 2 k T\right)\right\}^{-1}$

$C_{\mathrm{DL}}$ is known to be a pure capacity, independent of frequency at least up to frequencies of the order of $1 \mathrm{MHz}$ [21] (see Fig. $2 b$ for the equivalent circuit denved up to now). The second parallel component of the interface admittance, $-\mathrm{J} \omega \Delta \sigma_{0} / \Delta \psi_{0}$, does not originate from the electrostatic interaction between charge and potential, as is the case for $C_{D L}$, but represents the variation of surface charge on the insulator with the $\psi_{0}$ potential. The relation between $\sigma_{0}, \psi_{0}$ and $\mathrm{pH}$ has been studied elsewhere [12], where we have derived

$2.303\left(\mathrm{pH}_{\mathrm{pzc}}-p H\right)=q \psi_{0} / k T+\sinh ^{-1}\left[\sigma_{0} / \delta q N_{\mathrm{s}}\right]$

In this equation, $\mathrm{pH}_{\mathrm{pzc}}$ represents the solution $\mathrm{pH}$ which is required to make the insulator surface electrically neutral, as determined by the ratio of the equilibrium constants of the surface acid-base reactions; $\delta$, first introduced by Healy et al. [22], characterizes the reactivity of the surface; $N_{\mathrm{s}}$ is the number of reaction sites per unit area on the insulator surface. To obtain eqn. (12) it has been assumed that $\delta^{2} \ll 1$, 
which is the case for all usual insulators in silicon technology, in particular oxides such as $\mathrm{SiO}_{2}$ or $\mathrm{Al}_{2} \mathrm{O}_{3}$ [23].

The branch of $Z_{12}$ parallel to $C_{\mathrm{DL}}$ (see Fig. 1b) contains two series contributions, because

$(J \omega)^{-1} \frac{\Delta \psi_{0}}{\Delta \sigma_{0}}=\left.(j \omega)^{-1} \frac{\partial \psi_{0}}{\partial \sigma_{0}}\right|_{\mathrm{pH}}+\left.(j \omega)^{-1} \frac{\partial \psi_{0}}{\partial \mathrm{pH}}\right|_{\sigma_{0}} \frac{\Delta \mathrm{pH}}{\Delta \sigma_{0}}$

Evaluation of the partial derivatives from eqn. (12) implies the assumption that the equilibrium of surface reactions is always established in the experimental time-scale. This is supported by the available evidence about the speed with which $\psi_{0}$ responds to a pH step [24]. The first term of eqn. (13) is the capacitance associated with the adsorbed charge due to surface reactions with $\mathrm{H}^{+} / \mathrm{OH}^{-}$ions, which can be written

$C_{\mathrm{a}}^{-1}=-\mathrm{d} \psi_{0} / \mathrm{d} \sigma_{0}=\frac{k T}{\delta q^{2} N_{\mathrm{s}}}\left[1+\left(\frac{\sigma_{0}}{\delta q N_{\mathrm{s}}}\right)^{2}\right]^{-1 / 2}$

The second term of eqn. (13) is the Warburg impedance, corresponding to the variation of surface $\mathrm{pH}$ due to the ac signal applied. This impedance follows from standard theory:

$Z_{\mathrm{u}}=\frac{1}{J \omega}\left(\left.\frac{\partial \psi_{0}}{\partial \mathrm{pH}}\right|_{a_{0}}\right) \frac{\Delta \mathrm{pH}}{\Delta \sigma_{0}}=\frac{k T}{q^{2}(2 \omega)^{1 / 2}} \frac{1-J}{\left(\left[\mathrm{H}^{+}\right] D_{\mathrm{H}^{+2}}^{1 / 2}+\left[\mathrm{OH}^{-}\right] D_{\mathrm{OH}^{-}}^{1 / 2}\right)}$

Here $D_{\mathrm{H}^{+}}$and $D_{\mathrm{OH}^{-}}$are the diffusivities of $\mathrm{H}^{+}$and $\mathrm{OH}^{-}$ions in the electrolyte.

The complete equivalent circuit for the whole EIS structure must take account of two additional elements: the bulk resistance of the electrolyte $R_{\mathrm{b}}$ and the impedance of the reference electrode. Both of these depend on the particular experimental conditions that have been used, and add series contributions to the total impedance (see Fig. 2c).

The equivalent circuit denved here for the insulator/electrolyte interface is similar to that which describes specific adsorption of tons and molecules at the mercury/electrolyte interface, which can be treated as a special case of the theory of partial charge transfer $[25,26]$.

In the limit of zero frequency, $Z_{\text {ie }}$ reduces to a pure capacitance which can be written

$C_{1 \mathrm{O}}=\lim _{\omega \rightarrow 0}\left(\frac{Z_{1 e}^{-1}}{j \omega}\right)=C_{\mathrm{DL}}+C_{\mathrm{s}}=C_{\mathrm{DL}}\left[1+\beta\left(1+\left(\frac{\sigma_{0}}{\delta q N_{\mathrm{b}}}\right)^{2}\right)^{1 / 2}\right]$

where $\beta$, a parameter which characterizes the $\mathrm{pH}$ sensitivity of the surface, is defined as [12]

$\beta=\delta q^{2} N_{\varsigma} / k T C_{\mathrm{DL}}$

In general, $\beta$ depends on the solution concentration and $\mathrm{pH}$ through $C_{\mathrm{DL}}$ (eqns. $9-11$ and 17). However, in the limit of high ionc strength, $C_{D L}$ reduces to the Stern 
capacitance, and then $\beta$ becomes a parameter which depends only on the insulator.

We will use this limiting value of $\beta$ in what follows, i.e. a definition where $C_{\mathrm{DL}}$ is replaced by $C_{\text {stern }}$. The $\psi_{0} / \mathrm{pH}$ characteristics of an insulator can be used to determine $\beta$ experimentally. When the ionic strength of the electrolyte is low, eqn. (I 1$)$ must be used to relate $C_{\mathrm{DL}}$ to $C_{\text {stern }}$ before applying eqn. (16). Insulators whose $\beta$ is sufficiently large $(>10)$ have a practically Nernstian $\mathrm{pH}$ response. According to eqn. (16) their interface capacitance $C_{1 e 0}$ will also tend to be very large. In the limit of perfectly Nernstian behaviour, $C_{1 \text { ieo }}$ becomes infinite. This is easly understood. since in the case that $\psi_{0}$ only depends on the solution $\mathrm{pH}, \mathrm{d} \psi_{0}$, caused by an externally applied voltage, is zero. On the other hand, in the extreme of very unreactive surfaces, $\beta$ is nearly zero and the interface impedance is composed only of the double-layer capacitance.

To calculate the $\mathrm{pH}$ dependence of $C_{1 e 0}, \sigma_{0}$ must be known. This charge is the charge on the insulator surface due to the exchange of $\mathrm{H}^{+}$and $\mathrm{OH}^{-}$ions with the electrolyte, as described in the site-dissociation model $[8,12]$ In any experimental situation, the charge balance equation (2) must be applied to find the relation between $\sigma_{0}$ and $\sigma_{\mathrm{d}}$. This is complicated by the possible presence of a component in $Q_{\text {tot }}$ near the electrolyte whose influence on $V_{\mathrm{FB}}$ is difficult to detect. In the absence of further information on this subject, an assumption must be made; we will assume that the charges in the double layer are balanced, i.e. that $\sigma_{0}+\sigma_{\mathrm{d}}=0$. This hypothesis can be justufied in two cases: when $\sigma_{0}$ and $\sigma_{\mathrm{d}}$ are much larger than the other charges present in the system, or when a mechanism is present which allows the charge in the insulator to respond to the internal electric field. Possibilities for such a mechanism in $\mathrm{SiO}_{2}$ will be discussed below.

With the assumption $\sigma_{0}=-\sigma_{d}, \psi_{d}$ can be used to obtain $C_{\mathrm{DL}}$ from eqn. (I1) and $\sigma_{0}$ from (9), and $\sigma_{0}, \mathrm{pH}$ and $Z_{1 e}$ then follow from eqns. (10), (12), (14) and (15). Theoretical curves of $C_{1 c 0}$ calculated in this way are shown in Fig. 3 which represents the influence of surface reactivity $\beta$, in a $10^{-4} M$ solution where $Z_{1 c}$ will be high. For insulators such as $\mathrm{Al}_{2} \mathrm{O}_{3}$ [12], and perhaps also $\mathrm{Si}_{3} \mathrm{~N}_{4}, \beta$ will be at least equal to 3 or 4. Based on Fig. 3, we can expect that the surface impedance will be negligible in this case On the other hand, when $\beta$ is lower, as is the case for $\mathrm{SiO}_{2}$, the impedance increases sharply and becomes sensitive to $\beta$. Figure 4 shows theoretical curves at various ionic strengths using the parameters typical for $\mathrm{SiO}_{2}: \beta=0.14$ and $\mathrm{pH}_{\mathrm{pzc}}=$ 2.2 [12]. In all cases, a clear dependence on the $\mathrm{pH}$ can be seen, especially near $\mathrm{pH}_{\text {pzc* }}$

An effect that has been neglected here is the adsorption of counterions in the electrolyte, i e. all ions other than $\mathrm{H}^{+}$or $\mathrm{OH}^{-}$. Although it is well known that the surface reactions with $\mathrm{H}^{+}$and $\mathrm{OH}^{-}$ions mainly determine the interface potential, it is possible that highly charged surfaces attract ions of the opposite sign into the inner part of the double layer. This type of adsorption is expected to occur for $\mathrm{pH}$ values far from $\mathrm{pH}_{\mathrm{pzc}}$, and its presence on $\mathrm{SiO}_{2}$ has been confirmed by radiotracer measurements [27] and colloid chemical data [23]. It can be predicted in general terms how the theory of the impedance of the insulator/electrolyte interface must be expanded to take account of this effect. Since a new charge $\sigma_{\mathrm{ad}}$ is present, a third 


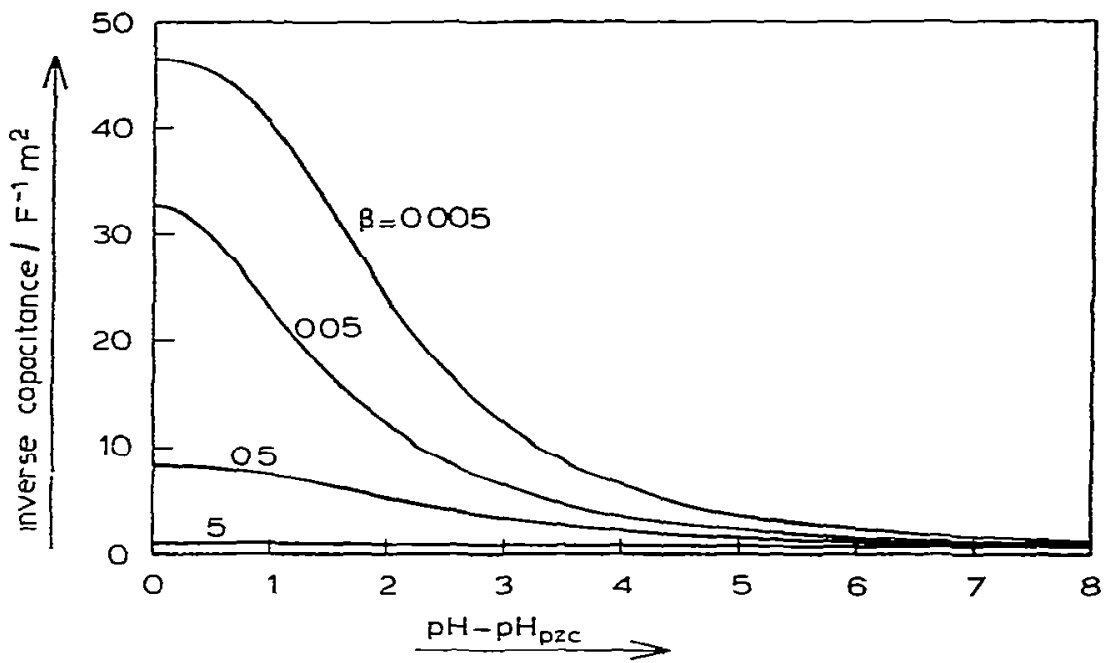

Fig 3. Theoretical curves showng the influence of the surface reactivity on the inverse interfacial capacitance $C_{1 e 0}^{-1}$ The values of $\beta$ are for high sonic strengths where $C_{\mathrm{DL}}=20 \mu \mathrm{F} \mathrm{cm}^{-2}$ Solution concentration is $10^{-5} \mathrm{M}$ and $C_{\text {stern }}=20 \mu \mathrm{F} \mathrm{cm}^{-2}$.

term $\Delta \sigma_{\mathrm{ad}} / \Delta \psi_{0}$ appears in eqn. (8). corresponding to a new parallel branch in the equivalent crrcuit. which will again be a capacitance in series with a Warburg impedance.

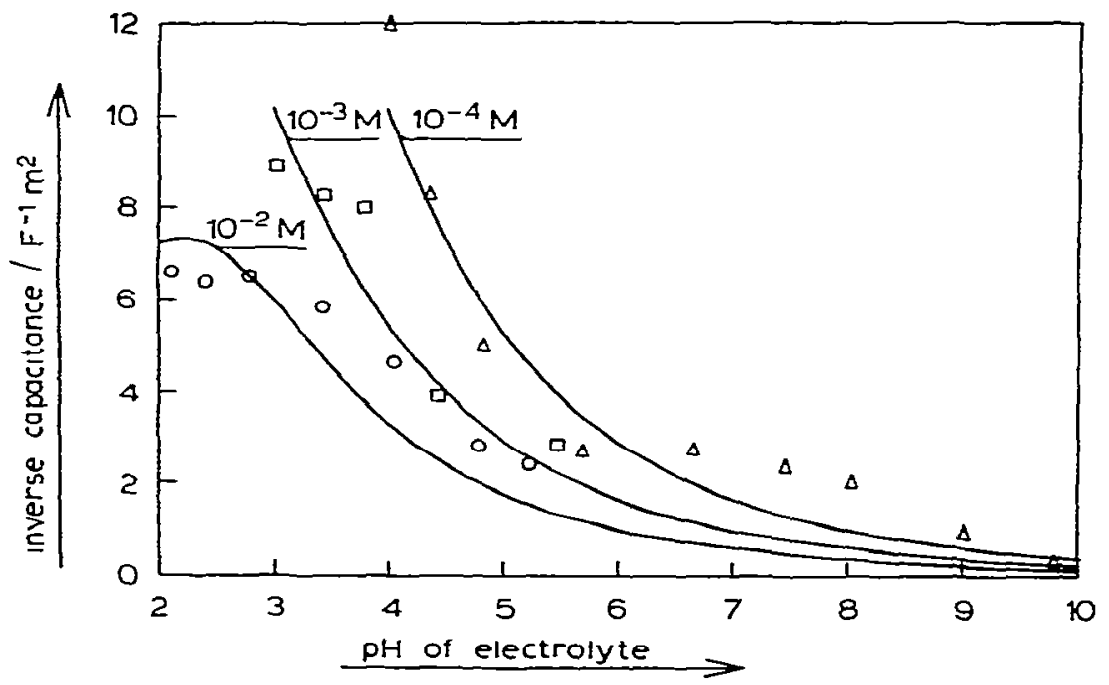

Fig. 4 Expenmental and theoretical inverse interfacial capacitance $C_{i e 0}^{-1}$ Expenmental points $(\Delta) 10^{-4}$ $M$ ( $\square$ ) $10^{-3} M,(O) 10^{-2} M$. Theoretical curves assume that $\beta=0.14$ in a $01 M$ solution, $C_{\mathrm{grern}}=20 \mu \mathrm{F}$ $c m^{-2}$ and $p H_{p z e}=22$ The value of $C_{i=0}^{-1}$ in the reference solution, $044 \mathrm{~F}^{-1} \mathrm{~m}^{2}$, has been subtracted 
In the region of $\mathrm{pH}$ values near $\mathrm{pH}_{\mathrm{pze}}$ and low ionic strengths where the $\mathrm{SiO}_{2} /$ electrolyte impedance is highest, it is known that no counterion adsorption can be detected by radiotracer techniques [27]. Only for $\mathrm{pH}$ values far from $\mathrm{pH} \mathrm{pzc}_{\mathrm{pzc}}$ and higher ionic strengths does adsorption appear to be present, but there the $\mathrm{SiO}_{2} /$ electrolyte impedance is much lower. It therefore seems legitimate to neglect counterion adsorption in the first-order theory of the insulator/electrolyte interface.

\section{EXPERIMENTAL}

\section{Sample preparation}

P-type silicon substrates with a (100) orientation were subjected to a standard cleaning procedure in fuming nitric acid and a $\mathrm{HF} / \mathrm{NH}_{4} \mathrm{~F}$ solution. This was followed by thermal oxidation in dry $\mathrm{O}_{2}$ at $1150^{\circ} \mathrm{C}$, and $30 \mathrm{~min}$ anneal in dry $\mathrm{N}_{2}$ at the same temperature. The resulting thickness of the oxide film was $102 \mathrm{~nm}$. The oxide on the back side was then etched away, and aluminium was evaporated on the back of the wafer. $A 30 \mathrm{~min}$ treatment in wet $\mathrm{N}_{2}$ at $450^{\circ} \mathrm{C}$ was used to anneal the back-side contact. A $1.8 \mu \mathrm{m}$ thick layer of Shipley AZ $1450 \mathrm{~J}$ photoresist on the front of the wafer was photolithographically patterned to define circular holes with a diameter of $2.5 \mathrm{~mm}$.

To expose the wafer to the electrolyte, a specially constructed glass cell was used, with a silicone rubber ring at the bottom which could be pressed on the wafer by a spring. Openings for the reference electrode, a glass electrode, and for introducing and extracting electrolytes are provided. With this arrangement, the photoresist layer defines the area of $\mathrm{SiO}_{2}$ which is in contact with the electrolyte, and the need to scribe the wafer in smaller portions is avorded.

Since very thin layers of $\mathrm{SiO}_{2}$ are required for the measurements, the wafer was etched through the photoresist mask in a $20: 1 \mathrm{NH}_{4} \mathrm{~F} / \mathrm{HF}$ solution to an oxide thickness of $12 \mathrm{~nm}$, as verified with an Applied Materials Model II ellipsometer.

\section{Materials}

All measurements were carried out in $\mathrm{NaCl}$ solutions to which $\mathrm{HCl}$ or $\mathrm{NaOH}$ at the same concentration was added to vary the $\mathrm{pH}$. The solutions were prepared from analytical reagent grade $\mathrm{NaCl}$ and $\mathrm{T}_{1}$ trisol $\mathrm{HCl}$ and $\mathrm{NaOH}$ solutions from Merck. Twice-distilled water was used throughout. Glassware, including the measurement cell, was cleaned with a solution of potassium dichromate in sulphuric acid, and then thoroughly nnsed. These precautions were designed to avoid possible contamination problems.

\section{Measurement set-up}

The complete cell was composed of the $\mathrm{Si} / \mathrm{SiO}_{2}$ sample, the electrolyte and a platinum electrode with an area of $1 \mathrm{~cm}^{2}$. The impedance of this electrode was 
measured with a second identical electrode in the same solutions as used for the subsequent measurements. In $10^{-4} \mathrm{M} \mathrm{HCl}$, the series capacitance of the Pt electrode was found to vary from $23 \mu \mathrm{F}$ at $1 \mathrm{~Hz}$ to $10 \mu \mathrm{F}$ at $20 \mathrm{~Hz}$. To avord $\mathrm{CO}_{2}$ contamination, the electrolyte in the cell was kept under a flow of pure $\mathrm{N}_{2}$. The ac admittance of the cell was measured by comparison with an accurately known reference impedance. using a P.A.R. 129A two-phase lock-in amplifier. The input signal had an amplitude of $20 \mathrm{mV}$, and a frequency which was vaned from 1 to 20 $\mathrm{Hz}$. The measured admittance of the cell was always transformed into a series-equivalent resistance and capacitance Reproducibility of this capacitance was in the order of $5 \times 10^{-4}$.

\section{Measurement procedure}

To eliminate the influence of the silicon surface impedance. the structure was biased in strong accumulation. A recorded $C-V$ curve was used to find the flat-band voltage, and the bias used was about $35 \mathrm{~V}$ below $V_{\mathrm{FB}}$. It was calculated that variations of $V_{\mathrm{FB}}$ only produce small variations in the capacitance of the silicon surface at this bias. Direct experimental confirmation, obtained by varying the applied voltage by $0.3 \mathrm{~V}$, produced a change in inverse capacitance of $0.8 \mathrm{~F}^{-1} \mathrm{~m}^{2}$. This represents an upper limit on the error due to $Z_{\mathrm{s}}$. The measurements were carned out at least three days after the sample was first exposed to an electrolyte

Considering the other components in the equivalent circuit of Fig. lc, the resistive effects of the bulk electrolyte, the bulk silicon, the $\mathrm{Pt}$ electrode and the oxide/ electrolyte interface are lumped together in the measured series resistance. and do not affect the capacitance. Finally, the oxide capacitance itself, which cannot of course be elıminated, must be known accurately. This requires a differential measurement, where two solutions were used. first, $0.1 \mathrm{M} \mathrm{NaCl}$ at $\mathrm{pH} 10$, for a minimal influence of $C_{\mathrm{Ie}}$, and second a solution at a lower concentration and a variable $\mathrm{pH}$. In this $w$ ay. the accuracy depends only on the reproducibility of the measurements.

The inverse of the interface capacitance then follows from the difference of the inverse capacitance in a given electrolyte, and the inverse capacitance in the $\mathrm{pH} 10$, $0.1 M$ reference solution. By this method inverse capacitances are determined, and therefore the expenmental and theoretical results are presented in this way.

\section{RESULTS}

\section{Measurements in the $10^{-1} \mathrm{M}$ solution}

The insulator thickness as determined from the capacitance in the $01 \mathrm{M} \mathrm{NaCl}$ solution at $\mathrm{pH}=10$ was $122 \mathrm{~nm}$ (assuming $\epsilon_{1}=341 \times 10^{-13} \mathrm{~F} \mathrm{~cm}^{-2}$ ), whereas an ellpsometric determination gave $12 \mathrm{~nm}$ Given the absolute accuracy involved in both methods, these two values agree with each other. In the course of the measurements, the measured capacitance increased by about $3 \%$, which was probably caused by a slow leakage of solution under the O-nng used for sealing. The large 
increase of capacitance which would occur if an 1onically conducting porous gel layer was formed, a mechanism proposed by some authors [28], is not observed.

The series resistance in the cell filled with this solution was small and frequencydependent, due to the influence of the series resistance of the platinum electrode. The frequency dependence of the capacitance was small, with a relatuve variation of $2 \times 10^{-3}$ in the frequency range $1-20 \mathrm{~Hz}$ which was considered.

\section{Measurements in $10^{-2}, 10^{-3}$ and $10^{-4}$ solutions}

Depending on the ionic strength a much larger electrolyte resistance $R_{\mathrm{b}}$ was present in these solutions, in particular for $10^{-4} M$ solutions. It was verified that the order of magnitude of $R_{\mathrm{b}}$ in $10^{-4} M$ electrolytes agreed with the measured electrolyte conductance and the geometry of the cell. A consequence is that only low frequencies will produce accurate values of the cell capacitance, since at high frequencies $R_{\mathrm{b}}$ dominates the measured total cell impedance. The only quaritity which can then be determined is $C_{\mathrm{se}}$, the zero-frequency limit of the interface capacitance. This was done by taking the difference of the inverse series capacitance measured at various $\mathrm{pH}$ values and ionic strengths, and the same measurement in the $0.1 M, \mathrm{pH} 10$ reference solution. For a given measurement frequency $f$, this procedure determines $C_{\mathrm{Ie}}$, the senes capacitance contribution to $Z_{\mathrm{ic}}$. Even at low frequencies, some frequency dependence remained in the value of $C_{1 e}\left(F_{1 g}\right.$. 5). The dependence was too large to be explained by the Warburg impedance $Z_{w}$ (eqn. 15), but was thought to be caused by the encapsulation procedure, which might not define perfectly sharp edges of the region exposed to the electrolyte, or possibly also

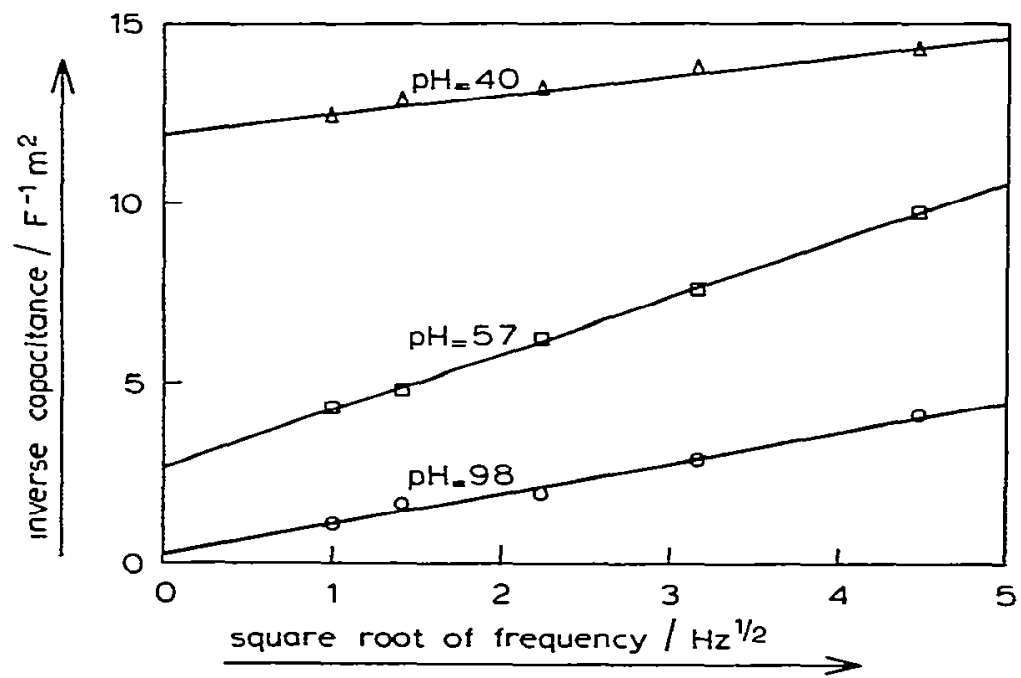

Fig 5 Measured frequency dependence of the inverse equvalent serses capacitance $C_{i e}^{-1}$ of the $\mathrm{S}_{1} \mathrm{O}_{2} /$ electrolyte interface in $10^{-4} M$ solutions 
by the cell geometry. This type of frequency dependence of the cell capacitance has been extensively discussed by de Leve [29], who showed that in the low-frequency limit it also behaves as a Warburg impedance, and depends on the ionic strength of the electrolyte. The procedure followed to determine $C_{\text {teo }}$ was to extrapolate all measurements of $f=0$ along the regression line for the $C_{1 \mathrm{t}} / \mathrm{f}^{1 / 2}$ relation. As expected, for the $10^{-3} M$ and $10^{-2} M$ solutions the $f^{1 / 2}$ dependence of $C_{2 c}$ was much smaller than for the $10^{-4} M$ solutions.

The leakage current flowng through the structure as a result of the applied voltage of $-35 \mathrm{~V}$ was found to be $<10^{-11} \mathrm{~A} \mathrm{~cm}^{-2}$.

In Fig. 4 the expermental results for $C_{1=0}$ obtained by extrapolation to zero frequency are shown for tonc strengths of $10^{-2} \mathrm{M}, 10^{-3} \mathrm{M}$ and $10^{-4} \mathrm{M}$. The t reoretical curves in the same figure are based on the values $\beta=0.14$ in $0.1 M$ sulutions. and $\mathrm{pH}_{\mathrm{pzc}}=2.2$ found in [12] for $\mathrm{SiO}_{2}$ surfaces. The quantitatuve agreement is reasonably good, especially since all parameters in the theory have been determined independently. It was found to be necessary to ensure that the measured capacitance was stable in time. since slow drifts sometımes occurred at low pH values. Also, some memory effect seemed to occur between two consecutive measurements at different $\mathrm{pH}$ values. whuch could be reduced by introducing a reference measurement in the $10^{-1} M$. pH 10 solution between two determinations.

\section{DISCUSSION}

The model of the interface impedance $Z_{12}$ presented here is simular to models used in the study of the impedance of metal/electrolyte electrodes [25,26], but it has not as yet appeared in the literature concerning electrolyte/insulator/silicon structure. A major difference between these two systems is that here no charge transfer occurs: therefore the case which most resembles the electrolyte/insulator interface is the adsorption of charged species at the $\mathrm{Hg} /$ electrolyte interface. The expression of the capacitance due to the insulator surface charge depends of course on the model used to expluin the formation of this charge. An early approach to this problem was made by Vermilyea [30] who used the theory of the adsorption of neutral substances at the $\mathrm{Hg} /$ electrolyte interface, as reviewed by Parsons [31], to explain the $\mathrm{pH}$ dependence of the capacitance of very thin anodic $\mathrm{Ta}_{2} \mathrm{O}_{5}$ films.

The model proposed by Yates et al. [8] is used here in the case that counterion adsorption can be neglected. This assumes that the interfacial potential drop $\psi_{0}$ occurs inside the electrolyte. In this way, the observed $\psi_{0} / \mathrm{pH}$ characteristics of insulators can be explained by a sensitivity parameter $\beta$. whose value can be predicted with existing data obtained from colloid dispersions [12]. The agreement found between the measurement results and the theoretical predictions supports the validity of this model. In particular, the curves at $10^{-4} M$ are dependent on $\beta$ (see $F_{1 g} 3$ ). and the experimental findings at this ionic strength agree with $\beta=014$ found previously [12]. The capacitance in the $10^{-2} M$ solution at $\mathrm{pH} 2$, on the other hand. depends strongly on the double-layer capacitance, since the $\mathrm{SiO}_{2}$ surface is very unreactive around its point of zero charge. The agreement found at $\mathrm{pH} 2$ supports the value $C_{\text {stern }}=20 \mu \mathrm{F} \mathrm{cm}{ }^{-2}$, obtained from ref. 8 . It is assumed here that 
the $\mathrm{SiO}_{2}$ surface is perfectly smooth. Silicon wafers of the type used here are chemically polished to extreme smoothness, and the resulting interface after thermal oxidation has been verified to have about $0.4 \mathrm{~nm}$ roughness [32].

The agreement between experiment and theory also indicates that the assumption $\sigma_{0}=-\sigma_{\mathrm{d}}$ is justified for $\mathrm{SiO}_{2}$ surfaces. It is easily verified that in the expenmental conditions described here, a charge of about $10^{-6} \mathrm{C} \mathrm{cm}^{-2}$ is induced by the applied dc voltage in the silicon substrate, and hence also in the region of the $\mathrm{SiO}_{2} /$ electrolyte interface. A model in wh ch this charge is incorporated in $\sigma_{0}$ would lead to interfacial impedances much lower than those observed. It must then be concluded that this charge resides inside the $\mathrm{SiO}_{2}$, probably near the interface with the electrolyte. The presence of $6 \times 10^{12}$ negative charges $\mathrm{cm}^{-2}$ inside the $\mathrm{SiO}_{2}$ layer is not an unreasonable concept; it could be considered, for example to be due to a number of buned $\mathrm{OH}$ groups which is much smaller than the density on the outer surface $\left(N_{\mathrm{s}} \approx 5 \times 10^{14}\right.$ sites $\left.\mathrm{cm}^{-2}\right)$. Using tritium exchange studies, Yates and Healy [33] have concluded that silica, heat treated at $800^{\circ} \mathrm{C}$ to make it non-porous, contains a considerable number of buned $\mathrm{OH}$ groups. A process for charge build-up which must be considered in this context is the injection of electrons into the $\mathrm{SiO}_{2}$ by the anodic applied voltage, as proposed by Niccolian et al. [34] They find that these electrons react with internal $\mathrm{OH}$ groups, which can enter $\mathrm{SiO}_{2}$ layers even at room temperature [35], to create a fixed negative space charge which counteracts the applied voltage. This negative space charge would then explain the very low leakage currents through $\mathrm{S}_{1} \mathrm{O}_{2}$ films under anodic potential and the fact that the double layer at the $\mathrm{SiO}_{2}$ /electrolyte interface is balanced, i.e. that $\sigma_{0}+\sigma_{\mathrm{d}}=0$. Another observation explained by the presence of this negative space charge is the slow dnft towards positive voltages of the flat-band voltage of $\mathrm{SiO}_{2}$-gate ISFETs, reported by Leistiko [19]. The possibility of electronic conduction through $\mathrm{SiO}_{2}$ layers has been discussed by Schmidt [36], Mornson and co-workers [37,38] and Revesz [39].

A few expenmental results concerning the influence of $\mathrm{pH}$ on the impedance of the oxıde/electrolyte interface for other oxides can be found in the literature of the anodic oxidation of metals such as aluminium and tantalum [30,40]. Libsch and Devereux [40] used $36 \mathrm{~nm} \mathrm{Al}{ }_{2} \mathrm{O}_{3}$ layers, which was too thick to see any influence of $\mathrm{pH}$; this is expected for materials with a high $\beta$. Vermilyea [30], however, measured capacitance variations on $2.4 \mathrm{~nm}$ layers of $\mathrm{Ta}_{2} \mathrm{O}_{5}$ with a maximum change at around $\mathrm{pH} 8$. For solutions of 001 to $2 M$ the inverse interfacial capacitance $C_{100}^{-1}$ at $\mathrm{pH} 8$ was in the order of $1 \mathrm{~F}^{-1} \mathrm{~m}^{2}$; the frequency dependence of Vermilyea's data makes it difficult to be more precise. This agrees well with the value to be expected for an oxide known to have a high $\beta$ [41], at least of the same order as for $\mathrm{Al}_{2} \mathrm{O}_{3}$. If we take $\beta=5-10$, and $C_{\mathrm{DL}}=C_{\text {stern }}=20 \mu \mathrm{F} \mathrm{cm} \mathrm{cm}^{-2}$ at high ionic strengths, the maximum $C_{1 e 0}^{-1}$ expected in this case can be seen from eqn. (16) to be $0.5-0.8 \mathrm{~F}^{-1} \mathrm{~m}^{2}$.

\section{CONCLUSIONS}

From the results presented here, the following conclusions can be drawn:

(l) The model of the oxide/electrolyte interface based on reactions of surface 
sites with $\mathrm{H}^{+}$and $\mathrm{OH}^{-}$ions provides an explanation for the measured $\mathrm{C}_{\mathrm{ie}}^{-1} / \mathrm{pH}$ curves for $\mathrm{SiO}_{2}$. The parameter values agree with those found in earlier $\psi_{0} / \mathrm{pH}$ measurements.

(2) The interfacial capacitance depends on the charge originating from the mechanism of $\mathrm{pH}$ response, and is different from the double-layer capacitance $C_{\mathrm{DL}}$. This means the oxide capacitance $C_{\mathrm{ox}}$ is the capacitance of the $\mathrm{SiO}_{2}$ layer from the $\mathrm{S} / / \mathrm{S}_{1} \mathrm{O}_{2}$ interface up to the region where the $\mathrm{pH}$ response mechanism is located. Therefore, the fact that this value of $C_{\mathrm{ox}}$ agrees with the capacitance expected from the ellipsometrically determined thickness implies that the $\mathrm{pH}$ response mechanism must be located at the $\mathrm{S}_{1} \mathrm{O}_{2}$ / electrolyte interface.

\section{ACKNOWLEDGEMENTS}

The authors wish to express their gratitude for the support recelved from the late Prof. Dr. G.A. Bootsma, who encouraged us with many discussions about this work H J M Geeraedts and the staff of the Solid-state Electronics Group assisted with the sample preparation. We thank $M$. Sluyters-Rehbach and $J$. Sluyters for providing a prepublication copy of ref. 26 One of us (L B ) thanks the Nationaal Fonds voor Wetenschappelijk Onderzoek (N.F.W.O) of Belgium for having awarded him a fellowship

\section{REFERENCES}

I R M Reeves in J O’M Bockris and B E Conways (Eds). Modern Aspects of Electrochemistry, Vol 9, Plenum Press, New York. 1974 p 239 see also ref 15

2 J H A Pieper and D A de Vooys, J Electroanal Chem, 53 (1974) 243

3 J Lyklema, Discuss Faraday Soc, 42 (1966) 81

4 R P Abendroth, J Collond Interface Scı, 34 (1970) 591

5 Y G Berube and P L de Bruyn, J Colloid Interface Sc1 . 27 (1968) 305

6 R J Hunter and H J L Wrght, J Colloid Interface Sc1, 37 (1971) 564

7 S Levine and A L. Smuth, Discuss Faraday Soc, 52 (1971) 290

8 DE Yates, S Levine, and T W Healy, J Chem Soc Faraday Trans 1, 70 (1974) 1807

9 P Bergveld, I E.E.E. Trans Biomed Eng., BME-19 (1972) 342

10 P Bergueld, N F de Rooy, and J N. Zemel. Nature, 273 (1978) 438

11 J Janata and R.J Huber, Ion Select. Electrode Rev, 1 (1979) 31

12 L. Bousse, N F de Roo1, and P. Bergueld, I E E E Trans Electron Devices, in press

13 N F de Rooij and $P$ Bergueld in ST Pantelides (Ed), The Physics of $\mathrm{S}_{1} \mathrm{O}_{2}$ and its Interfaces, Pergamon, New York. 1978, p 433

14 P R Barabash and R.S C Cobbold, I E.E.E Trans Electron Devices, ED.29 (1982) 102

15 MJ Sparnaay, The Electrical Double Layer, Pergamon. Oxford, 1972

16 T A.J Payens. Philips Res Rep. 10 (1955) 425

17 B E. Deal, J Electrochem Soc, 121 (1974) $198 \mathrm{C}$

18 L. Bousse, J. Chem Phys. 76 (1982) 5128.

19 O Leistiko, Phys Ser . 18 (1978) 445

20 S M. Sze, Physics of Semuconductor Devices, 2nd edn, Wiley, New York, 1981

21 R Parsons, in J O'M Bockns (Ed), Modern Aspects of Electrochemistry, Vol 1, Butterworths, London, 1954, p 103

22 T.W Healy, DE Yates, L.R Whute and D Chan, J Electroanal Chem, 80 (1977) 57 
23 J.A. Davis, R.O. James and J.O. Lecke, J. Collord Interface Sci, 63 (1978) 480.

24 L. Bousse, Thess, Twente Unversity of Technology, 1982.

$25 \mathrm{R}$. Parsons in P. Delahay (Ed). Advances in Electrochemistry and Electrochemical Engineering, Vol. 7, Wsley, New York, 1970, p. 177.

26 M. Sluyters-Rehbach and J H. Sluyters un J O'M. Bockns, B E. Conwray, and E. Yeager (Eds), Comprehensive Treatise on Electrochemustry, Vol 5, Plenum Press. New York, in press

27 W. Smit, C L.M Holten, H N Stein, J.J M de Goej and H M.J Theelen, J. Collond Interface Sci , 63 (1978) 120.

28 I R Lauks, I E.E.E. Trans Electron Devices, ED-26 (1979) 1952

$29 \mathrm{R}$ de Levie, J. Electroanal Chem, 9 (1965) 117.

30 D.A Vermilyea, Surf Sc, 2 (1964) 444

31 R. Parsons in P. Delahay (Ed), Advances in Electrochemistry and Electrochemical Enguneenng, Vol 1. Interscience, New York, 1961, p. 1

32 O L. Knvanek, T.T Sheng and D C Tsu, Appl Phys Lett, 32 (1978) 437

33 D E Yates and T.W. Healy, J. Collord Interface Sci, 55 (1976) 9.

34 E.H. Nicollan, C N. Berglund, P F. Schmidt and J M Andrews, J Appl Phys, 42 (1971) 5654

35 G.L. Holmberg, A B. Kuper and F D. Mirald, J. Electrochem Soc, 117 (1970) 677.

36 P F. Schmidt, J. Electrochem. Soc, 115 (1968) 167

37 M J Madou, K W. Frese and S R Morrison, Phys Stat Sol. (a), 57 (1980) 705

38 S E. Morrison, M.J Madou and K W Frese, Appl Surf Sci, 6 (1980) 138

39 A G Revesz, Phys Stai Sol, 24 (1967) 115

40 T.A Libsch and OF Devereux, J Electrochem Soc, 121 (1974) 400

41 T. Matsuo and M Esashi, Sensors and Actuators, 1 (1981) 77 